\title{
In vivo regulation by glutathione of methionine adenosyltransferase S-nitrosylation in rat liver
}

\author{
Fernando J. Corrales*, Félix Ruiz* and José M. Mato \\ Division of Hepatology and Gene Therapy, Department of Internal Medicine, School of Medicine, Universidad de Navarra, Pamplona, Spain
}

Background/Aims: Ethanol consumption and pathological conditions such as cirrhosis lead to a reduction of hepatic glutathione. Hepatic methionine adenosyltransferase, the enzyme that synthesizes $S$-adenosylmethionine, the major methylating agent, is regulated in vivo by glutathione levels. We have previously shown that nitric oxide inactivates methionine adenosyltransferase in vivo by S-nitrosylation. In this study, we aimed to investigate the regulation by glutathione of methionine adenosyltransferase S-nitrosylation in rat liver.

Methods: Rat hepatocytes and whole animals were treated with buthionine sulfoximine, an inhibitor of glutathione synthesis, and methionine adenosyltransferase S-nitrosylation and activity were determined.

Results: In hepatocytes, buthionine sulfoximine led to the S-nitrosylation and inactivation of methionine adenosyltransferase. Restoring glutathione levels in

$P^{n}$ ROTEIN S-nitrosylation - the addition and removal of nitric oxide (NO) (nitrogen monoxide) groups to cysteine residues - is emerging as a remarkably common switch to control protein function $(1,2)$. Thus, the activity of an increasing number of proteins, including transcription factors, membrane proteins and cytosolic enzymes, has been demonstrated to be regulated in vitro by S-nitrosylation $(1,2)$. Moreover, isolated rat hemoglobin (3), the canine ryanodine receptor (4) and rat liver methionine adenosyltransferase (5) (MAT, EC 2.5.1.6, also known as S-adenosylmethionine (Ad-

Received 12 March; revised 10 May; accepted 25 May 1999

Correspondence: José M. Mato, Division of Hepatology and Gene Therapy, Department of Internal Medicine, University of Navarra, 31008 Pamplona, Spain.

Tel: 34948 425678. Fax: 34948425677.

e-mail: jmmato@unav.es

*FJC and FR contributed equally to this work hepatocytes treated with buthionine sulfoximine, by the addition of glutathione monoethyl ester, a permeable derivative of glutathione, led to the denitrosylation and reactivation of methionine adenosyltransferase. In whole animals, buthionine sulfoximine led also to methionine adenosyltransferase S-nitrosylation and inactivation. S-Nitrosylation and inactivation of methionine adenosyltransferase induced by buthionine sulfoximine in whole animals was prevented by glutathione monoethyl ester.

Conclusions: These results indicate that in vivo hepatic methionine adenosyltransferase exists in two forms in equilibrium, nitrosylated (inactive) and denitrosylated (active), which are regulated by both the cellular levels of nitric oxide and glutathione.

Key words: Buthionine sulfoximine; Glutathione; Liver MAT; Nitric oxide; S-nitrosylation.

oMet) synthetase) have all been shown to be S-nitrosylated in vivo. However, little is known about the physiological mechanisms that govern protein S-nitrosylation in vivo or in intact cells. Target proteins may exist in two equilibrium forms - nitrosylated (SNO) and denitrosylated ( $\mathrm{SH})$-, and this equilibrium is thought to be regulated by both the cellular levels of NO donors and the concentration of reduced glutathione (GSH).

Hepatic MAT, a key enzyme of methionine metabolism that converts this amino acid into AdoMet, exists in two forms, which are a homotetramer (MAT I) and a homodimer (MAT III) of a single $\alpha$ subunit of $43.7 \mathrm{kDa}$ molecular weight (6-8). We have previously demonstrated that increased NO production in the liver, during septic shock, led to the S-nitrosylation and inactivation of hepatic MAT (5). We have further shown an inactivation of MAT in isolated rat hepatocytes by incubation with S-nitrosoglutathione monoethylester, an NO donor permeable through the 
plasma membrane (5). Moreover, MAT I and MAT III, purified from rat liver, are S-nitrosylated and inactivated by a variety of NO-donors (3-morpholinosydnonimine, S-nitroso- $\mathrm{N}$-acetylpenicillamine and S-nitrosoglutathione) $(5,9)$. The hepatic MAT $\alpha$ subunit contains 10 cysteine residues $(10,11)$. Inactivation of hepatic MAT by NO donors has been shown to be the result of S-nitrosylation of a single cysteine residue of the $\alpha$ subunit located in position 121 (9). In vitro, NOmediated hepatic MAT S-nitrosylation and inactivation was reversed by millimolar GSH (5). In the present study, we directly evaluated the effect of intracellular GSH on MAT III S-nitrosylation and activity in a model of rat hepatocytes treated with buthionine sulfoximine (BSO), a well-known inhibitor of GSH synthesis (12). We also report here on the role of GSH in the regulation of hepatic MAT III S-nitrosylation and activity in vivo.

\section{Materials and Methods \\ Materials}

Protease inhibitors (phenylmethylsulfonyl fluoride (PMSF) and benzamidine), ATP, L-methionine, DL-dithiothreitol, BSO and GSH were obtained from Sigma (St. Louis, MO, USA); $\left[2{ }^{3} \mathrm{H}\right]$ ATP $(25.5$ $\mathrm{Ci} / \mathrm{mmol}$ ) was from Amersham (Little Chalfton, UK). The cation exchanger, AG 50W-X4 (200 to 400 mesh), was from Bio-Rad Laboratories (Richmond, CA, USA). DEAE Sephacel and phenyl Sepharose were from Pharmacia (Uppsala, Sweden). 2-Mercaptoethanol was from Merck AG (Darmstadt, Germany), and scintillation fluid Optiphase Hisafe 3 was from Wallac (Loughborough, UK). High-performance liquid chromatography (HPLC) column Aminopropyl Spherisorb for glutathione determination was from Waters (Bedford, MA, USA). Glutathione monoethyl ester (EGSH) was synthesized as previously described (13).

\section{Isolation and incubation of hepatocytes}

Hepatocytes were isolated from normally fed Wistar albino rats $(250$ g) as described by Castaño et al. (14). Isolated hepatocytes were incubated as follows: 1 to $4 \mathrm{ml}$ of cell suspension $\left(2 \times 10^{6}\right.$ cells $\left./ \mathrm{ml}\right)$ were shaken in stopped $20-\mathrm{ml}$ vials at $37^{\circ} \mathrm{C}$ in the presence of $10 \mathrm{mM}$ glucose. The gas phase was $95 \% \mathrm{O}_{2}$ and $5 \% \mathrm{CO}_{2}$. After $30 \mathrm{~min}$ preincubation, BSO or buffer was added, and cells were incubated for the desired period of time. The suspension of hepatocytes was poured after the indicated periods into precooled, centrifuge tubes and immediatcly centrifuged for $10 \mathrm{~s}$. Cells were then washed twice in phosphate-buffered saline buffer before being homogenized by 3 cycles of freezing and thawing in $10 \mathrm{mM}$ Tris, $0.3 \mathrm{M}$ sucrose, and $1 \mathrm{mM}$ ethylene glycol-bis $(\beta$-aminoethyl ether)-N,N-tetraacetic acid (EGTA) (pH 7.5) containing $0.1 \%$ PMSF and $0.1 \%$ benzamidine. The cytosol was obtained by centrifugation at $100000 \mathrm{~g}$ at $4^{\circ} \mathrm{C}$. Cell viability was determined before and after the incubation by the Trypan blue exclusion test. MAT III activity and MAT III S-nitrosylation were analyzed in this last supernatant as described below. Only cell preparations with a viability greater than $85 \%$ were used. All animals were treated humanely, and study protocols were in compliance with our institution's guidelines for the use of laboratory animals.

\section{BSO treatment}

Animals received intraperitoneal injections of BSO $(4 \mathrm{mmol} / \mathrm{kg})$ or saline at 8 a.m. as previously reported (13). Samples were collected 2 h later, after the administration of BSO. Liver samples were removed and quickly freeze-clamped in liquid nitrogen. Livers were homogenized in 4 volumes of $10 \mathrm{mM}$ Tris $/ \mathrm{HCl}(\mathrm{pH} 7.5$ ), containing 0.3 $\mathrm{M}$ sucrose, $1 \mathrm{mM}$ EGTA, $0.1 \%$ PMSF, and $0.1 \%$ benzamidine. The homogenate was centrifuged for $20 \mathrm{~min}$ at $10000 \mathrm{~g}$ at $4^{\circ} \mathrm{C}$; the supernatant was again centrifuged for $1 \mathrm{~h}$ at $100000 \mathrm{~g}$. MAT III activity and MAT III S-nitrosylation were analyzed in this last supernatant as described below.

\section{GSH and oxidized glutathione measurements}

GSH and oxidized glutathione (GSSG) content was measured by high-performance liquid chromatography according to the method described by Reed et al. (15). Briefly, $200 \mu \mathrm{l}$ of the cytosolic fraction obtained as indicated above was immediately derivatized using 1fluoro-2,4-dinitrobenzene. Samples were then chromatographed on a 3-aminopropyl-Spherisorb, $20 \times 4.6 \mathrm{~mm}, 5 \mu \mathrm{m}$ HPLC column, equilibrated in $80 \%$ methanol. Elution was performed with $0.5 \mathrm{M}$ sodium acetate in $64 \%$ methanol and followed at $365 \mathrm{~nm}$. Analysis of the chromatogram was performed with Beckman System Gold software.

\section{Cytosolic nitrite and nitrate measurements}

Nitrite and nitrate were measured using a NOA 280 chemiluminiscence detector following the method recommended by Sievers (16). Cytosolic nitrite and nitrate were reduced to NO by incubation with glacial acetic acid containing $350 \mathrm{mM} \mathrm{INa}$ or $1 \mathrm{~N} \mathrm{HCl}$ containing $50 \mathrm{mM} \mathrm{VCl}$, respectively. The resulting NO was measured by the chemiluminescence derived from its reaction with ozone (16).

\section{Determination of MAT III S-nitrosylation and activity}

To measure the S-nitrosylation of MAT III in intact hepatocytes, the protein was purified from the cytosolic fraction of $4 \mathrm{ml}$ of cell suspension $\left(2 \times 10^{6}\right.$ cells $\left./ \mathrm{ml}\right)$ after incubation with the indicated reagents as described previously (5). Briefly, the cytosol was loaded onto a $2-\mathrm{ml}$ column of DEAE Sephacel equilibrated in a buffer containing $10 \mathrm{mM}$ HEPES, $10 \mathrm{mM} \mathrm{MgSO}$, and $1 \mathrm{mM}$ ethylendiaminetetracetic acid (EDTA) ( $\mathrm{pH} \mathrm{7.5)} \mathrm{(buffer} \mathrm{A).} \mathrm{The} \mathrm{column} \mathrm{was} \mathrm{washed} \mathrm{with} 10$ volumes of buffer A containing $100 \mathrm{mM} \mathrm{KCl}$ before elution with $2 \mathrm{ml}$ of buffer A containing $200 \mathrm{mM} \mathrm{KCl}$. The eluted enzyme was then chromatographed in a 1-ml phenyl Sepharose column equilibrated in buffer A with $200 \mathrm{mM} \mathrm{KCl}$, and then with 10 volumes of $10 \mathrm{mM}$ HEPES and $1 \mathrm{mM}$ EDTA (pH 7.5). Dimethyl sulfoxide (DMSO) is commonly used to elute MAT III from the phenyl Sepharose column (17). Because DMSO interfered with the NO detection, the resin containing MAT III protein was directly incubated with $2.2 \mathrm{mM} \mathrm{HgCl}$, and the amount of NO released was measured using a Sievers NOA 280 nitric oxide analyzer as described above. Standard curves were generated with known concentrations of S-nitrosoglutathione prepared according to Ruiz et al. (5). As previously reported (5), the purity of MAT III bound to the phenyl Sepharose column was greater than $90 \%$, as judged by sodium dodecyl sulfate-polyacrylamide gel electrophoresis. To determine the recovery of S-nitrosylated MAT III purified by this procedure, a sample of MAT III, purified from rat liver as described previously (11), was reacted in vitro with $100 \mu \mathrm{M}$ GSNO as described by Ruiz et al. (5), and the number of S-nitrosothiols (SNO) groups incorporated per milligram of protein was determined in an aliquot (5). The rest of the S-nitrosylated enzyme was added to a freshly prepared homogenate of hepatocytes. MAT III was then purified and the number of SNO groups was determined as mentioned above. The recovery of SNO groups into MAT III was between $80 \%$ to $90 \%$. To measure S-nitrosylation of MAT III in liver, the protein was purified from the cytosolic fraction of about $0.2 \mathrm{~g}$ of liver and the number of SNO groups incorporated per milligram of protein was determined as described above for hepatocytes. To measure the activity of MAT III in the cytosol derived from intact hepatocytes or liver samples, the enzyme was purified, as mentioned above, by DEAE Sephacel and phenyl Sepharose chromatography. The enzyme activity was then measured, as described by Cabrero et al. (18), by directly incubating the phenyl Sepharose resin containing MAT III with saturating concentrations of methionine and ATP (5 $\mathrm{mM}$ each) in the absence of any reducing agent. Total MAT activity was determined according to ibrero et al. (18) in the presence of saturating concentrations of $m$ :onine and ATP and in the absence of any reducing agent using the cytosolic extracts prepared as described above. 
Statistics

The data are the mean+SEM of at least three independent experiments. Statistical significance was estimated with Student's $t$-test. A $p$-value of less than 0.05 was considered significant.

\section{Results}

\section{$M A T S$-nitrosylation in isolated rat hepatocytes}

BSO is a well-known permeable inhibitor of GSH synthesis which inhibits the enzyme $\gamma$-glutamyl-cysteine synthetase, the first step in GSH synthesis from cysteine (12). We therefore tested the ability of BSO to reduce GSH levels in isolated rat hepatocytes and its cffect on MAT III S-nitrosylation and activity. As expected, the addition of $10 \mathrm{mM}$ BSO to isolated rat hepatocytes induced a time-dependent depletion of total intracellular glutathione (GSH plus GSSG) (Fig. 1). Thus, after 60 min incubation with BSO the intracellular levels of glutathione were reduced by about $50 \%$ as compared to control hepatocytes (Fig. 1). At the concentration of BSO used in the present experiments, this molecule had no effect on the ratio GSH/ GSSG (about 100) (data not shown). The latter is important since GSSG has been previously shown to in-

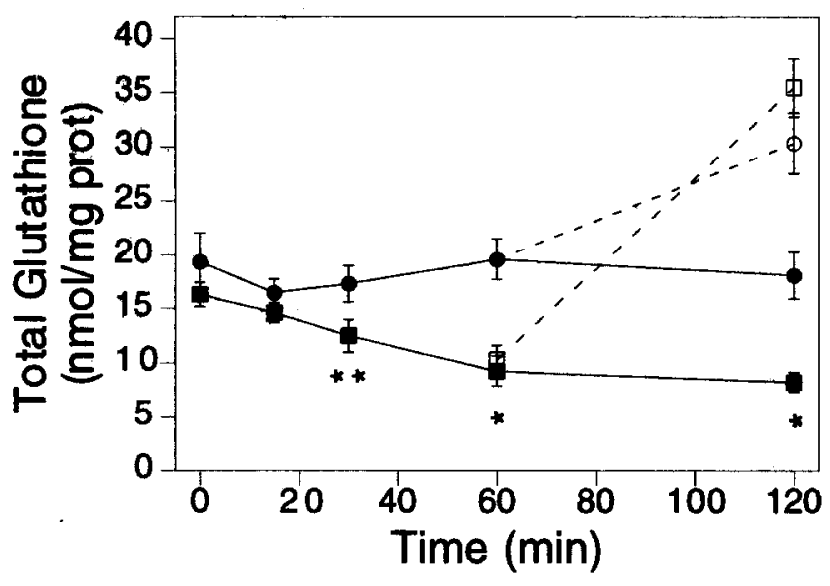

Fig. 1. Effect of D,L-buthionine S, R-sulfoximine (BSO) on glutathione levels in isolated rat hepatocytes. Isolated rat hepatocytes were incubated in the absence (0) or presence (a) of $10 \mathrm{mM}$ BSO for $60 \mathrm{~min}$. At the end of this period, $B S O$ was removed from the media by centrifugation, and, after washing cells twice, the hepatocytes were resuspended in fresh media and incubated again for another $60 \mathrm{~min}$ in the absence (0,O) or presence ( $\square, \square)$ of $10 \mathrm{mM} \mathrm{BSO}$ containing $5 \mathrm{mM}$ glutathione monoethylester (EGSH) $(\mathrm{O}, \square)$. Total glutathione was determined as described in Materials and Methods at the times indicated in the figure. Values are expressed as means $\pm S E M$ of three independent experiments in triplicate. At the indicated times, values in the presence of $B S O$ were significantly different from the corresponding control values in its absence ( ${ }^{*} \mathrm{p}<0.005$; $* * \mathrm{p}<0.05)$. hibit both MAT I and MAT III purified from rat liver (17).

The addition of BSO to isolated rat hepatocytes also induced a time-dependent intracellular accumulation of nitrite plus nitrate (NOx), the inactive metabolites of NO (Fig. 2). Thus, after $60 \mathrm{~min}$ in the presence of BSO the intracellular levels of NOx increased about 3fold (Fig. 2). BSO had no effect on the expression in hepatocytes of the inducible NO synthase (iNOS) gene or in the levels of iNOS protein, analyzed by Northern and Western blot, respectively (data not shown). No significant lysis of hepatocytes was observed after incubation with $10 \mathrm{mM}$ BSO under the present conditions (data not shown).

The addition of $10 \mathrm{mM}$ BSO to a suspension of isolated rat hepatocytes induced a time-dependent increase in the S-nitrosylation of MAT III (Fig. 3). Thus, after $60 \mathrm{~min}$ incubation in the presence of BSO the number of SNO groups incorporated into MAT III increased about 7-fold (about 2 sites per MAT subunit) with respect to cells incubated in the absence of BSO (Fig. 3). As shown in Fig. 4, BSO-mediated MAT III

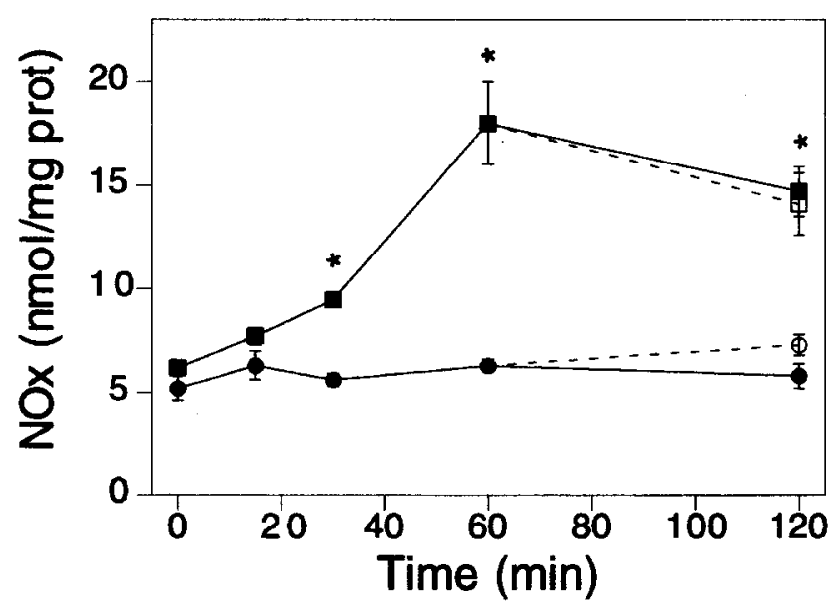

Fig. 2. Effect of D,L-buthionine $S, R$-sulfoximine (BSO) on nitrite and nitrate (NOx) levels in isolated rat hepatocytes. Isolated rat hepatocytes were incubated in the absence (O) or presence (1) of $10 \mathrm{mM} \mathrm{BSO}$ for $60 \mathrm{~min}$. At the end of this period, BSO was removed from the media by centrifugation, and, after washing cells twice, the hepatocytes were resuspended in fresh media and incubated again for another $60 \mathrm{~min}$ in the absence $(0,0)$ or presence $(\square, \square)$ of $10 \mathrm{mM}$ BSO containing $5 \mathrm{mM}$ glutathione monoethylester (EGSH) (O, $\square$ ). NOx levels were determined as described in Materials and Methods at the times indicated in the figure. Values are expressed as means $\pm S E M$ of three independent experiments in triplicate. At the indicated times (*) values in the presence of BSO were significantly different from the corresponding control values in its absence $(\mathrm{p}<0.001)$. 


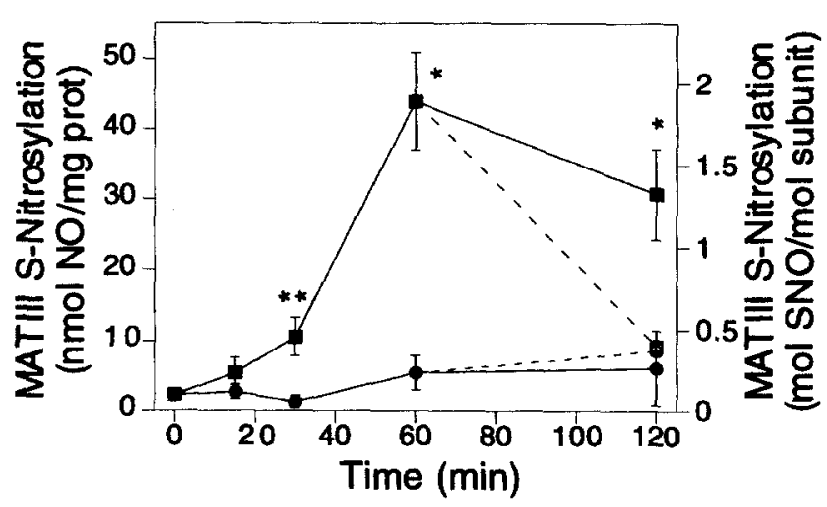

Fig. 3. Effect of $D, L$-buthionine $S, R$-sulfoximine ( $B S O$ ) on MAT III S-nitrosylation in isolated rat hepatocytes. Isolated rat hepatocytes were incubated in the absence (O) or presence (口) of $10 \mathrm{mM}$ BSO for $60 \mathrm{~min}$. At the end of this period, $B S O$ was removed from the media by centrifugation, and, after washing cells twice, the hepatocytes were resuspended in fresh media and incubated again for another 60 min in the absence $(0,0)$ or presence $(\square, \square)$ of $10 \mathrm{mM}$ BSO containing $5 \mathrm{mM}$ glutathione monoethylester (EGSH) (O, $\square$ ). Methionine adenosyltransferase (MAT) III S-nitrosylation was determined as described in $\mathrm{Ma}$ terials and Methods at the times indicated in the figure. Values are expressed as means $\pm S E M$ of three independent experiments in triplicate. At the indicated times, values in the presence of $B S O$ were significantly different from the corresponding control values in its absence $\left({ }^{*} \mathrm{p}<0.005\right.$; $* * \mathrm{p}<0.05$ ).

S-nitrosylation led to a reduction of the activity of the enzyme of about $80 \%$. When hepatocytes were incubated with $0.5 \mathrm{mM} \mathrm{N} \mathrm{N}^{\mathrm{G}}$-nitro-L-arginine (L-NNA), an inhibitor of NOS activity with higher affinity for the constitutive enzyme, the increase in the cellular content of NOx in response to BSO was abolished and, as expected, no effect of this agent on MAT III S-nitrosylation and inactivation was detected (data not shown). Incubation of hepatocytes with $0.5 \mathrm{mM} \mathrm{L-NNA}$ had no effect on the cellular depletion of GSH induced by BSO (data not shown).

To demonstrate that the effect of BSO on MAT III S-nitrosylation and activity was mediated by the depletion of intracellular GSH caused by this molecule, we tested the effect of restoring GSH levels on MAT III S-nitrosylation and activity in cells pre-treated with BSO. To restore cellular GSH levels, after being depleted by BSO administration, we used EGSH a wellknown permeable derivative of GSH, which, upon its entrance to the cell, is converted into GSH and ethanol (12). The addition of $5 \mathrm{mM}$ EGSH to isolated rat hepatocytes pre-treated for $60 \mathrm{~min}$ with $10 \mathrm{mM}$ BSO increased, within the following $60 \mathrm{~min}$, the intracellular

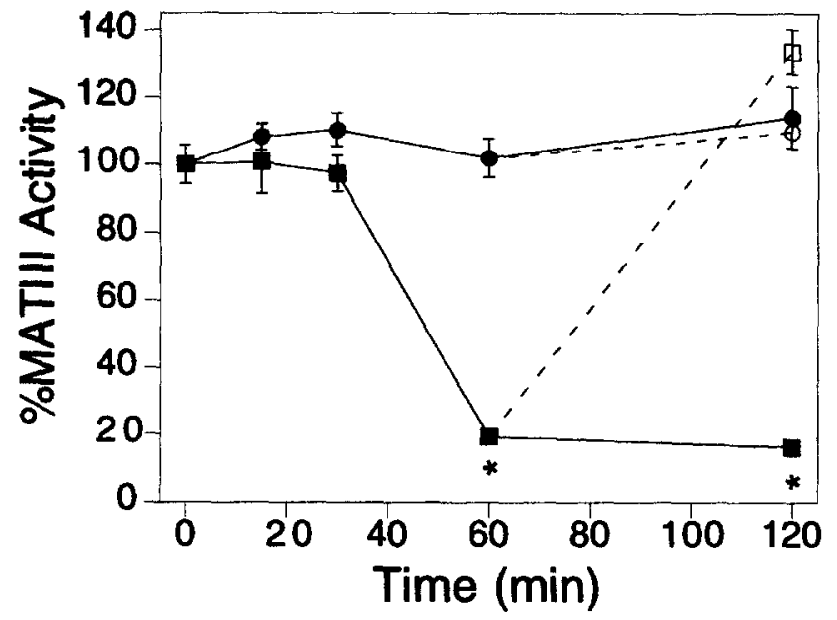

Fig. 4. Effect of $D, L$-buthionine $S, R$-sulfoximine (BSO) on methionine adenosyltransferase (MAT) III activity in isolated rat hepatocytes. Isolated rat hepatocytes were incubated in the absence (O) or presence (D) of $10 \mathrm{mM} \mathrm{BSO}$ for 60 min. At the end of this period, BSO was removed from the media by centrifugation, and, after washing cells twice, the hepatocytes were resuspended in fresh media and incubated again for another $60 \mathrm{~min}$ in the absence (O,O or presence ( $\square, \square$ ) of $10 \mathrm{mM} \mathrm{BSO}$ containing $5 \mathrm{mM} \mathrm{EGSH}$ (O, $\square$ ). MAT III activity was determined as described in Materials and Methods at the times indicated in the figure. $100 \%$ MAT activity was $40 \pm 4$ pmol/min/mg prot. Values are expressed as means $\pm S E M$ of three independent experiments in triplicate. At the indicated times (*) values in the presence of $B S O$ were significantly different from the corresponding control values in its absence $(\mathrm{p}<0.005)$.

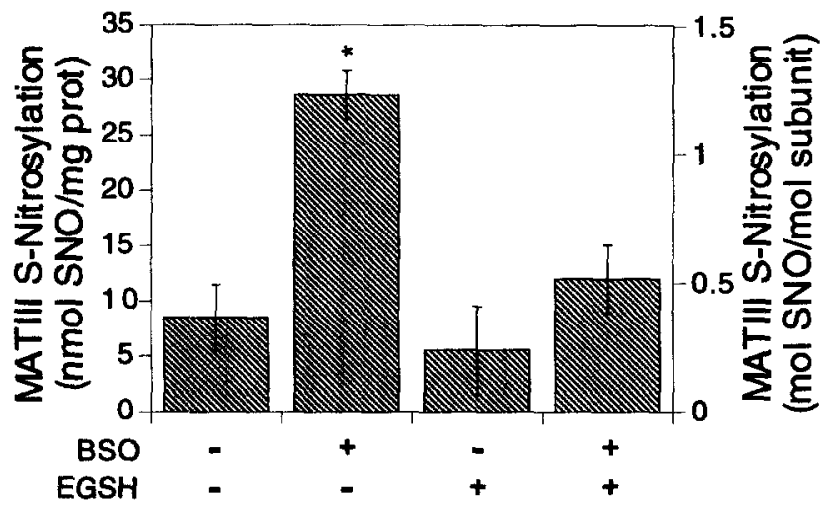

Fig. 5. Effect of D,L-buthionine S,R-sulfoximine (BSO) administration on rat liver methionine adenosyltransferase (MAT) III S-nitrosylation. Hepatic levels of MAT III Snitrosylation were determined $2 \mathrm{~h}$ after the intraperitoneal injection of BSO (4 mmollkg), BSO (4 mmollkg) plus glutathione monoethylester (EGSH) ( $7.5 \mathrm{mmol} / \mathrm{kg}$ ), EGSH (7.5 mmollkg) or saline as described in Materials and Methods. Values are expressed as means $\pm S E M$ of three independent experiments in triplicate. The value in the presence of BSO $\left(^{*}\right)$ was significantly different from the control $(\mathrm{p}<0.001)$. 


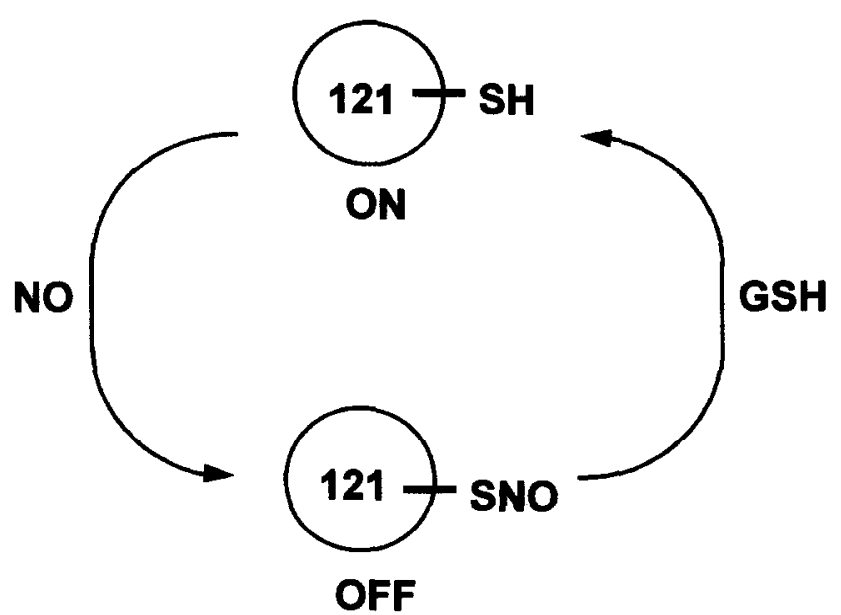

Fig. 6. A model for cysteine S-nitrosylation as a control switch to regulate hepatic methionine adenosyltransferase (MAT) activity. In this model, hepatic MAT $\alpha$ subunits exist in two forms in equilibrium, nitrosylated (SNO, inactive) and denitrosylated ( $\mathrm{SH}$, active), which are regulated by both the intracellular levels of nitric oxide (NO) and glutathione (GSH). Cysteine 121 is the residue of the hepatic $M A T \alpha$ subunit involved in the reaction with NO donors and GSH.

GSH levels to reach values comparable to those observed in control cells treated with the EGSH (Fig. 1). This increase in intracellular GSH resulted in loss of most of the NO groups incorporated into MAT III as a consequence of the addition of BSO (Fig. 3), and returned the activity of the enzyme to that of control hepatocytes (Fig. 4). These results indicate that MAT III S-nitrosylation in isolated rat hepatocytes is a reversible process regulated by both the intracellular concentration of NO donors and GSH, and that conditions that resulted in loss of SNO groups reversed the inactivation of the enzyme. Nitrite and nitrate (NOx) are the inactive end-products of NO metabolism. When EGSH was added to hepatocytes pretreated with BSO, the increase in the cellular content of NOx had already reached a plateau (Fig. 2), indicating that no further NO was generated by the hepatocytes in response to this agent. Consequently, the administration of EGSH to hepatocytes pre-treated with BSO had no effect on the cellular content of $\mathrm{NOx}$ (Fig. 2).

In vivo $S$-nitrosylation of hepatic MAT

Hepatic MAT activity is known to be regulated in vivo by GSH levels (13). We have previously demonstrated that the intraperitoneal injection of BSO to rats led to depletion of GSH and inactivation of hepatic MAT. We have therefore determined whether BSO-induced hepatic MAT inactivation in vivo was also mediated by MAT S-nitrosylation. In vivo S-nitrosylation of rat liver MAT III was analyzed $2 \mathrm{~h}$ after the intraperitoneal injection of BSO $(4 \mathrm{mmol} / \mathrm{kg})$. As previously reported (5), liver MAT III purified from control animals contained SNO groups ( $8 \mathrm{nmol}$ per milligram MAT subunit; about 0.4 sites per MAT subunit) (Fig. 5). Administration of BSO to rats induced a 3.4-fold increase in S-nitrosylation of MAT III (about 1.3 sites per MAT subunit) (Fig. 5). As previously reported (13), hepatic glutathione levels and total MAT activity decreased by about $60 \%$ and $50 \%$, respectively, in animals treated with BSO (data not shown). MAT III activity decreased also by about $50 \%$ in response to BSO treatment (data not shown). Under the present conditions, the hepatic ratio of GSH/GSSG did not change significantly with BSO injection (data not shown). No effect on hepatic or serum NOx levels was observed after BSO administration (data not shown). The simultaneous administration of BSO $(4 \mathrm{mmol} / \mathrm{kg})$ and EGSH $(7.5 \mathrm{mmol} / \mathrm{kg})$ prevented the effect of BSO on liver MAT III S-nitrosylation (Fig. 5). As previously reported (13), the simultaneous administration to rats of EGSH also prevented the effect of BSO on hepatic GSH levels and MAT activity (data not shown). These results demonstrate that also in vivo the inactivation of hepatic MAT induced by BSO is mediated by Snitrosylation of the enzyme.

\section{Discussion}

We have previously implicated NO as a regulator of liver MAT. Our data show an inactivation of hepatic MAT by NO produced during septic shock (9) or hypoxia (19), whereas inhibition of NO synthase, by $\mathrm{N}_{-}$ nitro-L-arginine-methylester, prevented hepatic MAT inactivation in response to hypoxia (19). We have further shown that incubation of isolated rat hepatocytes with S-nitrosoglutathione monoethylester induced a dose-dependent inactivation of MAT that was reversed by removing the NO donor from the cell suspension (5). Moreover, the activity of MAT I and MAT III, purified from rat liver, was inactivated by incubation with NO donors (3-morpholinosydnonimine, S-nitroso-N-acetylpenicillamine and S-nitrosoglutathione), a process that was reversed by GSH $(5,9)$. Recently we were able to demonstrate that S-nitrosylation of hepatic MAT led to inactivation of the enzyme, both in vitro and in vivo (5). Finally, inactivation of hepatic MAT by NO donors has been shown to be the result of S-nitrosylation of a cysteine residue of the $\alpha$ subunit located in position 121 (9).

The role of GSH in controlling hepatic MAT activity has been previously demonstrated. Thus, conditions 
that led to hepatic GSH depletion, like BSO (13) or carbon tetrachloride (20) in rats and alcoholic cirrhosis in humans (21), were accompanied by a marked reduction in hepatic MAT activity $(13,20,22,23)$. In the present study, we directly evaluated the role of GSH on MAT III S-nitrosylation and activity in isolated rat hepatocytes and in an in vivo rat model. Whereas MAT III can be readily isolated in the absence of dithiothreitol in the buffers used for purification to avoid the loss of SNO groups in the enzyme, by using the procedure mentioned in Materials and Methods, no method is yet available that permits the rapid isolation of MAT I. Moreover, when MAT I was purified by conventional procedures (17) the enzyme lost the SNO groups. Therefore, only the effect of GSH on MAT III S-nitrosylation was studied. The experimental model of isolated rat hepatocytes eliminates the influence of other organs and cells on liver responses. Using this model, we have observed an increased S-nitrosylation and inactivation of MAT III during BSO-mediated GSH depletion. Incubation of rat hepatocytes with BSO was also accompanied by a moderate increase in the cellular concentration of NOx that was not caused by an increase in iNOS protein levels, but probably by the activation of pre-existing NOS. Since the collagenase method used to isolate rat hepatocytes has been shown to induce the expression of iNOS $(24,25)$, the increase in NOx content induced by BSO may be due to the activation of this pre-existing iNOS or of any other class of pre-existing NOS. This hypothesis is supported by the finding that incubation of hepatocytes with the NOS inhibitor L-NNA prevented the increase in the cellular content of NOx induced by BSO and, consequently, prevented the S-nitrosylation and inactivation of MAT III induced by this agent. These results suggested that the increase in the production of NO and the decrease in GSH levels could be the cause of MAT III S-nitrosylation and inactivation. This point was further supported by the finding that the addition of EGSH to hepatocytes pre-treated with BSO restored GSH levels, removed most of the SNO groups incorporated into MAT III, and reactivated the enzyme. These results and our previous observations indicate that hepatic MAT $\alpha$ subunits exist in two forms in equilibrium, nitrosylated (inactive) and denitrosylated (active), which are regulated by both the cellular levels of NO and GSH (Fig. 6).

We had previously demonstrated that the administration of BSO to rats led to hepatic GSH depletion and MAT inactivation and that this effect in vivo of BSO could be prevented by the simultaneous administration of EGSH (13). Our present results show that in vivo, when rats were treated with $\mathrm{BSO}$, the reduction in
GSH levels induced by this molecule was enough to induce an increased S-nitrosylation of hepatic MAT III. This point was further supported by the finding that the addition of EGSH to rats prevented not only the effect of BSO on GSH levels and MAT activity (13), but also the increase in MAT III S-nitrosylation induced by this molecule. Whereas BSO induced a 7fold increase in MAT III S-nitrosylation in isolated rat hepatocytes, the magnitude of this effect in vivo was only 3.4-fold. These differences could be due to the finding that in isolated rat hepatocytes, but not in whole animals, BSO induced not only a depletion of GSH but also an increase in the cellular content of NOx. While the reason for these differences between the in vivo and in vitro experiments with respect to the production of NOx remains unclear, the results with whole animals show that the incorporation of NO groups into MAT can be increased by reducing the cellular levels of GSH without necessarily stimulating the synthesis of NO. This is possible if, as depicted in Fig. 6, the nitrosylated and denitrosylated forms of MAT are in an equilibrium regulated by both the cellular levels of NO donors and GSH. A consequence of this model is that NO does not need to exhaust the GSH pool to react with specific SH residues of proteins, but the reaction depends on the reactivities of the low-mass NO donors and the GSH content.

According to this model, in resting hepatocytes, at low concentrations of NO donors, the rate of addition of NO groups to MAT will be slower than the rate of denitrosylation of the enzyme driven by the high intracellular concentration of GSH (millimolar) and, as a consequence, the enzyme $\alpha$ subunits will be mainly in the denitrosylated form and therefore in their active conformation. If the cellular concentration of GSH decreases, as during the inhibition of its synthesis by BSO, the rate of MAT denitrosylation will also be reduced and a new steady state will be reached where more MAT $\alpha$ subunits will contain SNO groups, switching the enzyme to its inactive conformation. Restoring the basal GSH levels would increase the rate of removal of NO groups from MAT, switching the enzyme to the denitrosylated active form. Increased MAT S-nitrosylation and inactivation could also be reached after increasing the cellular concentration of NO donors but without affecting the GSH content, as during septic shock $(9,26,27)$, or by simultaneously increasing the synthesis of NO and reducing GSH levels, as during hypoxia $(19,28)$. In both cases, returning the GSH concentration and/or NO synthesis to pre-stimulation levels would result in the removal of NO groups from MAT $\alpha$ subunits and in the reactivation of the enzyme. 
The finding that a reduction in the hepatic GSH levels can lead to protein S-nitrosylation in the absence of increased NO synthesis is of general interest since a number of conditions and agents that induce hepatic GSH depletion, like ethanol consumption, apoptosis, paracetamol and carbon tetrachloride administration, and pathological conditions such as cirrhosis $(20,21,29-41)$, may lead to the S-nitrosylation of a variety of proteins, in addition to MAT, thus contributing to the physiopathological effect of these various conditions and agents.

\section{Acknowledgements}

We thank Prof. Manuel Martín Lomas and Dr. Dacil Zurita for the synthesis of EGSH.

This work was supported by grants from the Plan Nacional de I+D (SAF 96/0108, SAF 98/0132), Europharma and Knoll. F.R. was supported by the Instituto de Coopcración Ibcroamericana.

\section{References}

1. Stamler JS. Redox signaling: nitrosylation and related target interactions of nitric oxide. Cell 1994; 78: 931-36.

2. Stamler JS, Toone EJ, Lipton SA, Sucher NJ. (S)NO signals: translocation, regulation, and a consensus motif. Neuron 1997; 18: 691-6.

3. Stamler J, Jia L, Eu J, McMahon T, Demchenko I, Bonaventura $\mathrm{J}$, et al. Blood flow regulation by S-nitrosohemoglobin in the physiological oxygen gradient. Science 1997; 276: 2034-7.

4. Xu L, Eu JP, Meissner G, Stamler JS. Activation of the cardiac calcium release channel (ryanodine receptor) by poly-S-nitrosylation. Science 1998; 279: 234-7.

5. Ruiz F, Corrales FJ, Miqueo C, Mato JM. Nitric oxide inactivates hepatic methionine adenosiltransferase in vivo by S-nitrosylation. Hepatology 1998; 28: 1051-7.

6. Kotb M, Mudd SH, Mato JM, Geller AM, Kredich NM, Chou JY, et al. Consensus nomenclature for the mammalian methionine adenosyltransferase genes and gene products. Trends in Genetics 1997; 13: 51-2.

7. Kotb M, Geller AM. Methionine adenosyltransferase: structure and function. Pharmacol Ther 1993; 59: 125-43.

8. Mato JM, Alvarez L, Ortiz P, Pajares MA. S-adenosylmethionine synthesys: molecular mechanisms and clinical implications. Pharmacol Ther 1997; 73: 1-16.

9. Avila MA, Mingorance J, Martinez-Chantar ML, Casado M, Martín-Sanz P, Boscá L, et al. Regulation of rat liver S-adenosylmethionine synthetase during septic shock: rolc of nitric oxidc. Hepatology 1997; 25: 391-6.

10. Horikawa $\mathrm{S}$, Ishikawa $\mathrm{M}$, Ozasa $\mathrm{H}$, Tsukada $\mathrm{K}$. Isolation of a cDNA encoding the rat liver S-adenosylmethionine synthetase. Eur J Biochem 1989; 184: 497-501.

11. Mingorance J, Alvarez L, Sánchez-Góngora E, Mato JM, Pajares MA. Site-directed mutagenesis of rat liver $S$-adenosylmethionine synthetase. Biochem J 1996; 315: 761-6.

12. Griffith $O$, Meister A. Potent and specific inhibition of glutathione synthesis by buthionine sulfoximine (S-n-butyl homocysteine sulfoximine). J Biol Chem 1979; 254: 7558-60.

13. Corrales F, Ochoa P, Rivas C, Martín-Lomas M, Mato JM, Pajares MA. Inhibition of glutathione synthesis in the liver leads to S-adenosyl-L-methionine synthetase reduction. Hepatology 1991; 14: 528-33.

14. Castaño JG, Alemany S, Nieto A, Mato JM. Activation of phos- pholipid methyltransferase by glucagon in rat hepatocytes. J Biol Chem 1980; 255: 9041-3.

15. Reed DJ, Babson JR, Beatty PW, Brodie AE, Ellis WW, Potter DW. High-performance liquid chromatography analysis of nanomole levels of glutathione, glutathione disulfide, and related thiols and disulfides. Anal Biochem 1980; 106: 55-62.

16. Sievers Nitric Oxide Analyzer NOA280. Operation and Service Manual. Boulder, CO. USA: Sievers Instruments I; 1996.

17. Pajares MA, Durán C, Corrales F, Pliego MM, Mato JM. Modulation of rat liver S-adenosylmethionine synthetase activity by glutathione. J Biol Chem 1992; 267: 17598-605.

18. Cabrero C, Puerta J, Alemany S. Purification and comparison of two forms of S-adenosyl-Lmethionine synthetase from rat liver. Eur J Biochem 1987; 170: 299-304.

19. Avila MA, Carretero MV, Rodriguez EN, Mato JM. Regulation by hypoxia of methionine adenosyltransferase activity and gene expression in rat hepatocytes. Gastroenterology 1998; 114: 364 71.

20. Corrales F, Giménez A, Alvarez L, Caballería J, Pajares MA, Andreu $\mathbf{H}$, et al. $\mathrm{S}$-adenosylmethionine treatment prevents carbon tetrachloride-induced S-adenosylmethionine synthetase inactivation and attenuates liver injury. Hepatology 1992; 16: 1022-7.

21. Vendemiale G, Altomare E, Trizio T, Le Grazie C, Di Padova C, Salerno M, et al. Effects of oral S-adenosyl-L-methionine on hepatic glutathione in patients with liver disease. Scand $\mathbf{J}$ Gastroenterol 1989; 24: 407-15.

22. Martín-Duce A, Ortiz P, Cabrero C, Mato JM. S-adenosyl-Lmethionine synthetase and phospholipid methyltransferase are inhibited in human cirrhosis. Hepatology 1988; 8: 65-8.

23. Cabrero C, Martín-Duce A, Ortiz P, Alemany S, Mato JM. Specific loss of the high-molecular-weight form of S-adenosyl-L-methionine synthetase in human liver cirrhosis. Hepatology 1988; 8 : $1530-4$.

24. Wang H, Gao X, Fukumoto S, Tademoto $S$, Sato $K$, Hirai $K$. Post-isolation inducible nitric oxide synthase gene expression due to collagenase buffer perfusion and characterization of the gene regulation in primary cultured murine hepatocytes. J Biochem (Tokyo) 1998; 124: 892-9.

25. López-Garcia MP. Endogenous nitric oxide is responsible of the early loss of P450 in cultured rat hepatocytes. FEBS Lett 1998; 438: $145-9$.

26. Billiar TR, Curran RD, Ferrari FK, Williams DL, Simmons RL. Kupffer cell: hepatocyte cocultures release nitric oxide in response to bacterial endotoxin. J Surg Res 1990; 48: 349-53.

27. Knowles RG, Salter M, Brooks SL, Moncada S. Anti-inflammatory glucocorticoids inhibit the induction by endotoxin of nitric oxide synthase in the lung, liver and aorta of the rat. Biochem Biophys Res Commun 1990; 172: 1042-8.

28. Shan X, Aw T, Shapira R, Jones D. Oxygen dependence of glutathione synthesis in hepatocytes. Toxicol Appl Pharmacol 1989; 101: 261-70.

29. Comporti M, Benedetti A, Chieli E. Studies on in vitro peroxidation of liver lipids in ethanol-treated rats. Lipids 1973; 8: 498502.

30. Chawla R, Lewis F, Kutner M, Bate D, Roy R, Rudman D. Plasma cysteine, cystine, and glutathione in cirrhosis. Gastroenterology 1984; 87: 770-6.

31. Lauterburg B, Davies S, Mitchell J. Ethanol suppresses hepatic glutathione synthesis in rats in vivo. J Pharmacol Exp Ther 1984; 230: 7-11.

32. Videla L, Iturriaga $H$, Pino $M$, Bunout $D$, Valenzuela $A$, Ugarte $\mathrm{G}$. Content of hepatic reduced glutathione in chronic alcoholic patients: influence of the length of abstinence and liver necrosis. Clin Sci 1984; 66: 283-90.

33. Speisky H, MacDonald A, Giles G, Orrego H, Israel Y. Increased loss and decreased synthesis of hepatic glutathione after acute ethanol administration. Turnover studies. Biochem J 1985; 225: $565-72$

34. Feo F, Pascale R, Garcea R, Daino L, Pirisi L, Frassetto S, et al. Effect of the variations of S-adenosyl-L-methionine liver content 
on fat accumulation and ethanol metabolism in ethanol-intoxicated rats. Toxicol Appl Pharmacol 1986; 83: 331-41.

35. Fernandez-Checa J, Ookhtens $\mathbf{M}$, Kaplowitz N. Effects of chronic ethanol feeding on rat hepatocytic glutathione. Relationship of cytosolic glutathione to efflux and mitochondrial sequestration. J Clin Invest 1989; 83: 1247-52.

36. Kaneo Y, Fujihara Y, Tanaka T, Kozawa Y, Mori H, Iguchi S. Effects of glutathione, as the dextran conjugate, on acetaminophen-induced hepatotoxicity. Chem Pharm Bull (Tokyo) 1989; 37: $218-20$.

37. Lieber C, Casini A, DeCarli L, Kim C, Lowe N, Sasaki R, et al. S-adenosyl-L-methionine attenuates alcohol-induced liver injury in the baboon. Hepatology 1990; 11: 165-72.
38. Wong B, Corcoran GB. N-Acetylcysteine stereoisomers as in vivo probes of the role of glutathione in drug detoxification. In: Viña J, editor. Glutathione: metabolism and physiological functions. Boca Raton, FL: CRC Press; 1990: 256-62.

39. Bray G, Tredger J, Williams R. S-adenosylmethionine protects against acetaminophen hepatotoxicity in two mouse models. Hepatology 1992; 15: 297-301.

40. Slater A, Stefan C, Nobel I, van den Dobbelsteen D, Orrenius S. Signalling mechanisms and oxidative stress in apoptosis. Toxicol Lett 1995; 82: 149-53.

41. Voehringer DW, McConkey DJ, McDonnell TJ, Brisbay S, Meyn RE. Bcl-2 expression causes redistribution of glutathione to the nucleus. Proc Natl Acad Sci USA 1998; 95: 2956-90. 